\title{
Nuclear IR SED of Type 2 Seyfert Galaxies
}

\author{
L. Videla ${ }^{1}$, P. Lira ${ }^{1}$, A. Alonso-Herrero ${ }^{2}$, \\ D. M. Alexander ${ }^{3}$, and M. Ward ${ }^{3}$ \\ ${ }^{1}$ Astronomy Department, University of Chile, Santiago, Chile \\ ${ }^{2}$ DAMIR, IEM, CSIC, Spain \\ ${ }^{3}$ Department of Physics, Durham University, Durham, UK
}

Keywords. galaxies: Seyfert, infrared: galaxies.

We obtained deep images for 49 Seyfert 2 galaxies in 6 infrared bands: $J, H, K, L, M$, and $N$ with the VLT at Paranal, the NTT at La Silla, and Gemini at Pachón, to compile their nuclear infrared (IR) spectral energy distributions (SEDs). These galaxies were extracted from the Extended $12 \mu \mathrm{m}$ Galaxy Sample (Rush et al. 1993).

The deconvolution of the surface brightness profiles of the galaxies into a combination of a nuclear source and a stellar component (disk+bulge+bar, when needed) allows us to construct the nuclear IR SED. We use real seeing conditions and exclude local features like arms, rings or starburst regions. The mean spatial resolution is $\sim 250 \mathrm{pc}$.
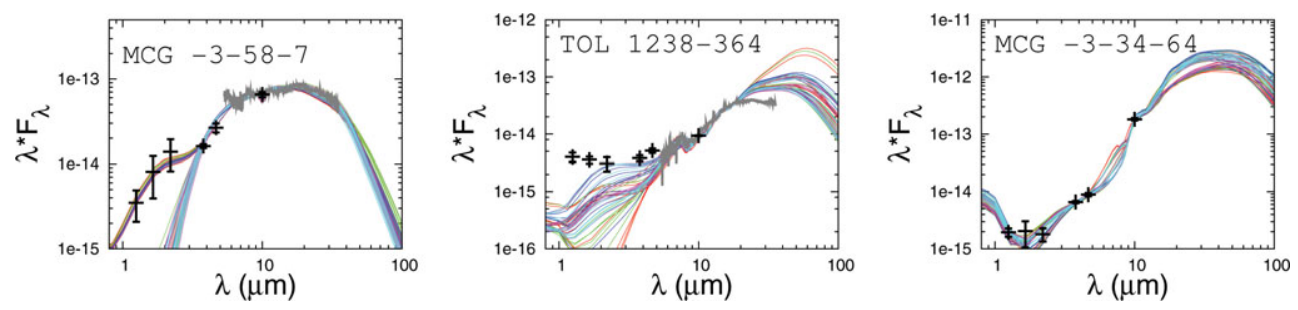

Figure 1. Examples of nuclear IR SEDs fitted using clumpy emission models. Left: MCG-3-58-7. Middle: TOL 1238-364. Right: MCG-3-34-64.

The nuclear SEDs were fitted using theoretical clumpy models (Nenkova et al. 2008), with examples shown in Figure 1. Our preliminary conclusion is that the preferred values for the radial scale of the torus are rather small (consistent with all available interferometric observations of the nearest AGN) although sizes as large as $R_{\text {out }} / R_{\text {in }}=200$ are also quite common. The average viewing angle corresponds to highly obscured objects. The optical depth has a very sharp maximum at $\tau_{V}=10$. We also conclude that the a number of clouds is between $N_{0}=5_{-}^{-}$ 15 , with the maximum centered at $N_{0}=12$, and opening angles are rather large (up to $75^{\circ}$ ).

Complementary Spitzer IRS spectroscopy covering 5-35 $\mu \mathrm{m}$ (Wu et al. 2009) will be included in future analysis (Videla et al., in preparation). The nuclear IR SED will allow us to statistically study the torus, which is the cornerstone of the unified model (Antonucci 1993).

\section{References}

Antonucci, R. 1993, ARAA, 31, 473

Nenkova, M., Sirocky, M. M., Nikutta, R., Ivezić, Ž., \& Elitzur, M. 2008, ApJ, 685, 160

Nenkova, M., Sirocky, M. M., Ivezić, Ž., \& Elitzur, M. 2008, ApJ, 685, 147

Rush, B., Malkan, M. A., \& Spinoglio, L. 1993, ApJS, 89, 1

Wu, Y., Charmandaris, V., Huang, J., Spinoglio, L., \& Tommasin, S. 2009, ApJ, 701, 658 